\title{
Neutron capture measurements on the s-process termination isotopes lead and bismuth
}

César Domingo Pardo*1,2, U. Abbondanno ${ }^{3}$, G. Aerts $^{4}$, H. Álvarez-Pol ${ }^{5}$, F. Alvarez-Velarde ${ }^{6}$, S. Andriamonje ${ }^{4}$, J. Andrzejewski ${ }^{7}$, P. Assimakopoulos ${ }^{8}$, L. Audouin ${ }^{1}$, G. Badurek ${ }^{9}$, P. Baumann ${ }^{10}$, F. Bečváŕr ${ }^{11}$, E. Berthoumieux ${ }^{4}$, S. Bisterzo ${ }^{12,1}$, F. Calviño ${ }^{13}$, D. Cano-Ott ${ }^{6}$, R. Capote $^{14,15}$, C. Carrapiço ${ }^{16}$, P. Cennini ${ }^{17}$, V. Chepel ${ }^{18}$, E. Chiaveri ${ }^{17}$, N. Colonna ${ }^{19}$, G. Cortes ${ }^{13}$, A. Couture ${ }^{20}$, J. Cox ${ }^{20}$, M. Dahlfors ${ }^{17}$, S. David ${ }^{21}$, I. Dillman ${ }^{1}$, R. Dolfini ${ }^{22}$, W. Dridi ${ }^{4}$, I. Duran ${ }^{5}$, C. Eleftheriadis ${ }^{23}$, M. Embid-Segura ${ }^{6}$, L. Ferrant ${ }^{21}$, A. Ferrari ${ }^{17}$,

R. Ferreira-Marques ${ }^{18}$, L. Fitzpatrick ${ }^{17}$, H. Frais-Koelbl ${ }^{14}$, K. Fujii ${ }^{3}$, W. Furman ${ }^{24}$, R. Gallino ${ }^{12}$, I. Goncalves ${ }^{18}$, E. Gonzalez-Romero ${ }^{6}$, A. Goverdovski ${ }^{25}$, F. Gramegna ${ }^{26}$, E. Griesmayer ${ }^{14}$, C. Guerrero ${ }^{6}$, F. Gunsing ${ }^{4}$, B. Haas $^{27}$, R. Haight ${ }^{28}$, M. Heil ${ }^{1}$, A. Herrera-Martinez ${ }^{17}$, M. Igashira ${ }^{29}$, S. Isaev ${ }^{21}$, E. Jericha ${ }^{9}$, Y. Kadi ${ }^{17}$, F. Käppeler ${ }^{1}$, D. Karamanis ${ }^{8}$, D. Karadimos ${ }^{8}$, M. Kerveno, ${ }^{10}$, V. Ketlerov ${ }^{25,17}$, P. Koehler ${ }^{30}$, V. Konovalov ${ }^{24,17}$, E. Kossionides ${ }^{31}$, M. Krtička ${ }^{11}$, C. Lamboudis ${ }^{8}$, H. Leeb ${ }^{9}$, A. Lindote ${ }^{18}$, I. Lopes ${ }^{18}$, M. Lozano ${ }^{15}$, S. Lukic ${ }^{10}$, J. Marganiec ${ }^{7}$, S. Marrone ${ }^{19}$, P. Mastinu ${ }^{26}$, A. Mengoni ${ }^{14,17}$, P.M. Milazzo ${ }^{3}$, C. Moreau ${ }^{3}$, M. Mosconi ${ }^{1}$, F. Neves ${ }^{18}$, H. Oberhummer ${ }^{9}$, M. Oshima ${ }^{32}$, S. O'Brien ${ }^{20}$, J. Pancin ${ }^{4}$, C. Papachristodoulou ${ }^{8}$, C. Papadopoulos ${ }^{33}$, C. Paradela ${ }^{5}$, N. Patronis ${ }^{8}$, A. Pavlik ${ }^{34}$, P. Pavlopoulos ${ }^{35}$, L. Perrot ${ }^{4}$, R. Plag ${ }^{1}$, A. Plompen ${ }^{36}$, A. Plukis ${ }^{4}$, A. Poch ${ }^{13}$, C. Pretel ${ }^{13}$, J. Quesada ${ }^{15}$, T. Rauscher ${ }^{37}$, R. Reifarth ${ }^{28}$, M. Rosetti ${ }^{38}$, C. Rubbia ${ }^{22}$, G. Rudolf ${ }^{10}$, P. Rullhusen ${ }^{36}$, J. Salgado ${ }^{16}$, L. Sarchiapone ${ }^{17}$, I. Savvidis ${ }^{23}$, C. Stephan ${ }^{21}$, G. Tagliente ${ }^{19}$, J.L. Tain ${ }^{2}$, L. Tassan-Got ${ }^{21}$, L. Tavora ${ }^{16}$, R. Terlizzi $^{19}$, G. Vannini ${ }^{39}$, P. Vaz ${ }^{16}$, A. Ventura ${ }^{38}$, D. Villamarin ${ }^{6}$, M. C. Vincente ${ }^{6}$, V. Vlachoudis $^{17}$, R. Vlastou ${ }^{33}$, F. Voss ${ }^{1}$, S. Walter ${ }^{1}$, H. Wendler ${ }^{17}$, M. Wiescher ${ }^{20}$, K. Wisshak ${ }^{1}$

The $\mathbf{n}$ _TOF Collaboration 
${ }^{1}$ Forschungszentrum Karlsruhe GmbH (FZK), Institut für Kernphysik, Germany; ${ }^{2}$ Instituto de Física Corpuscular, CSIC-Universidad de Valencia, Spain; ${ }^{3}$ Istituto Nazionale di Fisica Nucleare, Trieste, Italy, ${ }^{4}$ CEA/Saclay - DSM, Gif-sur-Yvette, France,${ }^{5}$ Universidade de Santiago de Compostela, Spain; ${ }^{6}$ Centro de Investigaciones Energeticas Medioambientales y Technologicas, Madrid, Spain, ${ }^{7}$ University of Lodz, Lodz, Poland, ${ }^{8}$ University of Ioannina, Greece $;{ }^{9}$ Atominstitut der Österreichischen Universitäten,Technische Universität Wien, Austria;

${ }^{10}$ Centre National de la Recherche Scientifique/IN2P3 - IReS, Strasbourg, France $;{ }^{11}$ Charles University, Prague, Czech Republic; ${ }^{12}$ Dipartimento di Fisica Generale, Università di Torino, Italy, ${ }^{13}$ Universitat Politecnica de Catalunya, Barcelona, Spain $;{ }^{14}$ International Atomic Energy Agency, NAPC-Nuclear Data Section, Vienna, Austria, ${ }^{15}$ Universidad de Sevilla, Spain;

${ }^{16}$ Instituto Tecnológico e Nuclear(ITN), Lisbon, Portugal; ${ }^{17}$ CERN, Geneva, Switzerland; ${ }^{18}$ LIP Coimbra \& Departamento de Fisica da Universidade de Coimbra, Portugal; ${ }^{19}$ Istituto Nazionale di Fisica Nucleare, Bari, Italy; ${ }^{20}$ University of Notre Dame, Notre Dame, USA $;{ }^{21}$ Centre National de la Recherche Scientifique/IN2P3 - IPN, Orsay, France, ${ }^{22}$ Università degli Studi Pavia, Pavia, Italy, ${ }^{23}$ Aristotle University of Thessaloniki, Greece, ${ }^{24}$ Joint Institute for Nuclear Research, Frank Laboratory of Neutron Physics, Dubna, Russia; ${ }^{25}$ Institute of Physics and Power Engineering, Kaluga region, Obninsk, Russia ${ }^{26}$ Istituto Nazionale di Fisica Nucleare(INFN), Laboratori Nazionali di Legnaro, Italy; ${ }^{27}$ Centre National de la Recherche Scientifique/IN2P3 - CENBG, Bordeaux, France, ${ }^{28}$ Los Alamos National Laboratory, New Mexico, USA $;{ }^{29}$ Tokyo Institute of Technology, Tokyo, Japan, ${ }^{30}$ Oak Ridge National Laboratory, Physics Division, Oak Ridge, USA ${ }^{31}$ NCSR, Athens, Greece; ${ }^{32}$ Japan Atomic Energy Research Institute, Tokai-mura, Japan;

${ }^{33}$ National Technical University of Athens, Greece, ${ }^{34}$ Institut für Isotopenforschung und Kernphysik, Universität Wien, Austria, ${ }^{35}$ Pôle Universitaire Léonard de Vinci, Paris La Défense, France, ${ }^{36}$ CEC-JRC-IRMM, Geel, Belgium; ${ }^{37}$ Department of Physics and Astronomy - University of Basel, Basel, Switzerland; ${ }^{38}$ ENEA, Bologna, Italy; ${ }^{39}$ Dipartimento di Fisica, Università di Bologna, and Sezione INFN di Bologna, Italy.

E-mail: cesar.domingo.pardo@cern.ch

Resonance cross sections relevant for the termination of the $s$-process reaction path have been determined for ${ }^{204,206,207} \mathrm{~Pb}$ and ${ }^{209} \mathrm{Bi}$ at the CERN neutron time-of-flight spectrometer $\mathrm{n}$-TOF. The measurements were carried out in the neutron energy range from $1 \mathrm{eV}$ up to $500 \mathrm{keV}$. By using a system of $\mathrm{C}_{6} \mathrm{D}_{6}$-detectors with optimized neutron sensitivity, the main corrections of previous measurements related to backgrounds from scattered neutrons could be practically eliminated. Other corrections were thoroughly treated by control measurements with additional samples for determination of the ambient background and of background from in-beam $\gamma$-rays as well as by detailed analyses via Monte Carlo simulations. The Maxwellian averaged cross sections of the four isotopes and their implications for the $s$-process abundance contributions in the $\mathrm{Pb} / \mathrm{Bi}$ region are discussed.

International Symposium on Nuclear Astrophysics - Nuclei in the Cosmos - IX June 25-30 2006

CERN, Geneva, Switzerland

\footnotetext{
*Speaker.
} 


\section{Introduction}

The isotopic abundances of the stable elements between iron and bismuth are essentially produced by the slow neutron capture process ( $s$ process) and the rapid neutron capture process $(r$ process). The $s$ process, which has been found to operate in thermally pulsing low mass asymptotic giant branch (AGB) stars is producing most of the $\mathrm{Pb} / \mathrm{Bi}$ abundances in stars of low metallicity [1,2]. Bismuth is the last isotope produced by the $s$ process because further neutron capture on Bi leads to the Po-isotopes, which are $\alpha$-unstable. The abundance pattern in this termination region is complex because of the branchings due to $\alpha$-recycling at ${ }^{210} \mathrm{Po}\left(\mathrm{t}_{1 / 2}=138 \mathrm{~d}\right)$ and at ${ }^{210 m} \mathrm{Bi}\left(\mathrm{t}_{1 / 2}=3 \mathrm{My}\right)$. The final $\mathrm{Pb} / \mathrm{Bi}$ abundance distribution is influenced by these branchings, which are sensitive to the neutron exposure at the $s$-process site. Since ${ }^{204} \mathrm{~Pb}$ is shielded from the $r$-process by its mercury isobar ${ }^{204} \mathrm{Hg}$, the observed ${ }^{204} \mathrm{~Pb}$ abundance is only produced by the $s$-process branching at ${ }^{204} \mathrm{Tl}$, which is very sensitive to the stellar temperature. Hence, the ${ }^{204} \mathrm{~Pb}$ abundance is determined by the strong temperature and neutron density variations characteristic of the thermal pulses in AGB stars. In addition, a fraction of the ${ }^{206,207,208} \mathrm{~Pb}$ abundances is produced by the decay of the long-lived uranium and thorium isotopes. By means of accurate neutron capture cross section measurements and using an appropriate stellar model, the s-process abundances $\left(N_{s}\right)$ of the $\mathrm{Pb} / \mathrm{Bi}$ isotopes can be more reliably determined. These $N_{s}$ values contain information about the aspects mentioned above, and allows one for a better decomposition of the respective $r$-process abundances and of the radiogenic contributions due to the $\mathrm{Th} / \mathrm{U} \alpha$ decays as well. The latter information is important to consolidate the reliability of the $\mathrm{Th} / \mathrm{U}$ cosmochronometer.

\section{Neutron capture measurements}

Radiative neutron capture measurements on ${ }^{204,206,207} \mathrm{~Pb}$ and ${ }^{209} \mathrm{Bi}$ were carried out at the CERN n_TOF installation using small and highly isotopically enriched samples (see Table 1). The small sample thicknesses led to correspondingly small corrections for neutron multiple scattering in the sample, self-shielding and $\gamma$-ray absorption effects. Even though some tiny resonances could not be observed due to the lower reaction rate of the thin samples, the main resonances which completely dominate the cross section in the relevant energy range for stellar nucleosynthesis (5$30 \mathrm{keV}$ ) could be measured in this way with improved systematic accuracy.

\begin{tabular}{ccc}
\hline Isotope & Enrichment (\%) & Thickness (atoms/barn) \\
\hline \hline${ }^{204} \mathrm{~Pb}$ & 99.71 & 0.00376 \\
${ }^{206} \mathrm{~Pb}$ & 99.76 & 0.0073 \\
${ }^{207} \mathrm{~Pb}$ & 92.4 & 0.0074 \\
${ }^{209} \mathrm{Bi}$ & 100 & 0.0173 \\
\hline
\end{tabular}

Table 1: Enrichment and thickness of the samples measured at $n_{-}$TOF.

The resolved resonance region is measured at $\mathrm{n} \_$TOF with excellent time of flight (TOF) resolution due to the $185 \mathrm{~m}$ flight path length and the short proton pulse width (6 ns rms) used in the neutron spallation source. More details about the facility can be found in Ref. [3]. A further 
advantage in this experiment is the broad neutron energy range covered, between $1 \mathrm{eV}$ and $1 \mathrm{MeV}$. It allowed us to measure resonances, which could not be observed in previous capture experiments due to a lower neutron energy cutoff at $2.5 \mathrm{keV}$ [4].

The samples were placed inside an evacuated sample changer made of carbon fibre. The detection system consisted of two neutron sensitivity optimized $\mathrm{C}_{6} \mathrm{D}_{6}$ detectors [5] placed at $\sim 125^{\circ}$ with respect to the incident neutron beam direction in order to minimize the angular distribution effects of the prompt $\gamma$-rays emitted after neutron capture. Details about the experimental setup at n_TOF can be found in Ref. [6]. Due to the low neutron sensitivity of the detectors and the setup in general, the TOF dependent background due to neutrons being scattered in the sample was only noticeable for one of the measured resonances in the bismuth sample, and negligible for all the rest. The correction needed in this exceptional case was also much smaller $(3.5 \%)$ than previous corrections in other experiments $(\sim 50 \%)[7,8]$.

The neutron fluence across the sample, $\Phi_{n}\left(E_{n}\right)$, was monitored by means of a thin ${ }^{6} \mathrm{Li}$ foil and four Si-detectors placed outside the neutron beam. The dependence of $\Phi_{n}$ on the neutron energy $E_{n}$ could be accurately determined by measuring the well known fission yields of ${ }^{235} \mathrm{U}$ and ${ }^{238} \mathrm{U}$ with a fission chamber [9]. A thin gold sample was measured in regular intervals in order to perform an absolute yield calibration by applying the so called saturated resonance technique [10].

The common dead-time due to the electronic processing of the signals from the detectors has been avoided in the present measurement because all events are recorded with Flash Analog to Digital Converters (Flash-ADC) [11]. The capture data was stored on tape and hard disk. The relevant parameters for the capture data analysis were extracted off-line by applying a pulse shape analysis routine.

\section{Capture data analysis and results}

The $\mathrm{C}_{6} \mathrm{D}_{6}$ detectors usually register, at most, one of the cascade $\gamma$-rays. Applying the pulse height weighting technique (PHWT)[12], the capture yield can be written as,

$$
Y\left(E_{n}\right)=f_{A u}^{s a t} \times \frac{N_{\gamma}^{w}\left(E_{n}\right)}{E_{c} \times \Phi_{n}\left(E_{n}\right)},
$$

where $N_{\gamma}^{w}$ is the weighted count rate for a certain TOF or neutron energy $E_{n}$. The weighting factors (WF) were calculated by means of detailed Monte Carlo (MC) simulations of the experimental setup following the procedure described in Refs. $[6,13,14]$. An uncertainty of less than $0.5 \%$ was estimated for the calculated WFs. $E_{c}$ is the neutron capture energy, $S_{n}+E_{n} \times A /(A+1)$, $S_{n}$ being the neutron separation energy. The yield calibration factor $f_{A u}^{s a t}$ is determined from the measurement of a gold reference sample (see previous section). Nevertheless, in order to achieve an uncertainty in the yield determination of $\lesssim 3 \%$ one needs to take into account additional systematic effects, which are mainly related to $i$ ) the (digital or electronic) threshold in the signals of the $\mathrm{C}_{6} \mathrm{D}_{6}$ detectors $\left(f^{t}\right)$ [14] and to ii) the angular distribution effects in the case of $p$-and $d$-wave resonances of low multiplicity $\left(f^{\theta}\right)$ [15]. The corrected capture yield is then more accurately given by,

$$
Y^{\prime}\left(E_{n}\right)=f^{\theta} \times f^{t} \times Y\left(E_{n}\right)
$$


For the $\mathrm{Pb}$ and $\mathrm{Bi}$ isotopes analyzed here, $f^{t}$ ranges between 1.031(3) and 1.070(3) depending on the particular isotope, the threshold level used in the experiment, and the spin and parity $\left(J^{\pi}\right)$ of each resonance. Angular distribution effects were only relevant in the measurement of ${ }^{206,207} \mathrm{~Pb}$, where the capture state decays by emission of only one or two $\gamma$-rays. Even though the detectors were placed at $125^{\circ}$, the solid angle covered by both detectors led to yield correction factors $\left(f^{\theta}\right)$ of up to $10 \%$ for some of the measured resonances. In most cases the factor $f^{\theta}$ could be accurately determined using previously measured decay patterns and angular distributions $[16,17,18,19]$.

Using the SAMMY code [20] an R-matrix analysis of the capture yield $Y^{\prime}$ was carried out. The background in this analysis could be best determined from the (n, $\gamma$ ) measurement of a $99.86 \%$ enriched ${ }^{208} \mathrm{~Pb}$ sample, which showed very few resonances in the entire neutron energy range. From the latter it was concluded that neutron induced backgrounds were negligible, whereas the main background contribution was due to in-beam $\gamma$-rays being scattered in the sample and registered in the $\mathrm{C}_{6} \mathrm{D}_{6}$ scintillators. This $\gamma$-ray scattering effect is identical for all $\mathrm{Pb}$ samples and this holds in very good approximation also for the bismuth measurement. The uncertainty in the cross section of the resolved resonance region due to the background analysis was estimated to be $1.5 \%$ for very broad resonances $(\Gamma>1 \mathrm{keV})$ but less than $0.5 \%$ in all other cases.

In the measurement of ${ }^{204} \mathrm{~Pb}$ the unresolved resonance region between $E_{n}=100 \mathrm{keV}$ and $E_{n}=400 \mathrm{keV}$ was analyzed using the FITACS code implemented in SAMMY. The so determined average capture cross section is important for deriving the Maxwellian average cross section (MACS) at thermal energies of $k T=20-30 \mathrm{keV}$.

The measured cross sections were convoluted with a Maxwell-Boltzmann distribution in order to obtain the relevant values for stellar nucleosynthesis calculations. These results are compared in Fig. 1 with the cross sections reported in Ref. [21]. The first remarkable aspect of the present measurements is the improvement in the uncertainty of the cross sections, usually more than a factor of two. This improvement is also translated into a more precise determination of the stellar $s$-process abundances (see later Sec. 4). The agreement with previous MACS [21] within the quoted uncertainties shows that the latter have been realistically evaluated in the past, except in the case of ${ }^{204} \mathrm{~Pb}$ where the MACS at $k T=5 \mathrm{keV}$ is now $35 \%$ higher due to three previously unobserved resonances [22]. In the case of ${ }^{206} \mathrm{~Pb}$ (dashed line in Fig. 1) our MACS is still preliminary mainly because of some resonances that have not been corrected yet for angular distribution effects.

\section{Implications in astrophysics}

$\mathrm{The} \mathrm{Pb} / \mathrm{Bi}$ isotopes are essentially synthesized in asymptotic giant branch (AGB) stars of low mass and low metallicity where most of the neutron exposure is due to the ${ }^{13} \mathrm{C}(\alpha, n){ }^{16} \mathrm{O}$ source, which operates at temperatures of about $10^{8} \mathrm{~K}(k T=8 \mathrm{keV})$. During the He shell flashes the ${ }^{22} \mathrm{Ne}(\alpha, n){ }^{25} \mathrm{Mg}$ reaction provides an additional neutron exposure at the higher thermal energy of $k T=23 \mathrm{keV}$. Since the latter irradiation is rather weak, the abundances of isotopes with small cross sections like ${ }^{206,207} \mathrm{~Pb}$ and mainly ${ }^{209} \mathrm{Bi}$ (see Fig. 1) is dominated by what has been produced during the interpulse phase at $k T=8 \mathrm{keV}$. Because of its much higher cross section, the situation for ${ }^{204} \mathrm{~Pb}$ is the opposite (Fig. 1) and the MACS in the higher thermal energy region ( $k T \sim 23 \mathrm{keV}$ ) is more relevant in this case. 


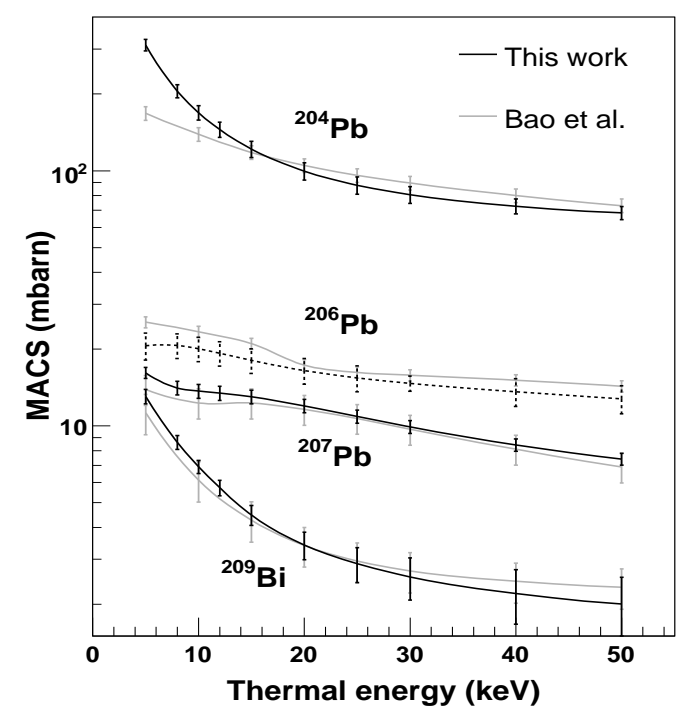

Figure 1: Maxwellian averaged cross sections for the isotopes measured at n_TOF compared with the cross sections from Bao et al. [21]. Note that the values for ${ }^{206} \mathrm{~Pb}$ are still preliminary.

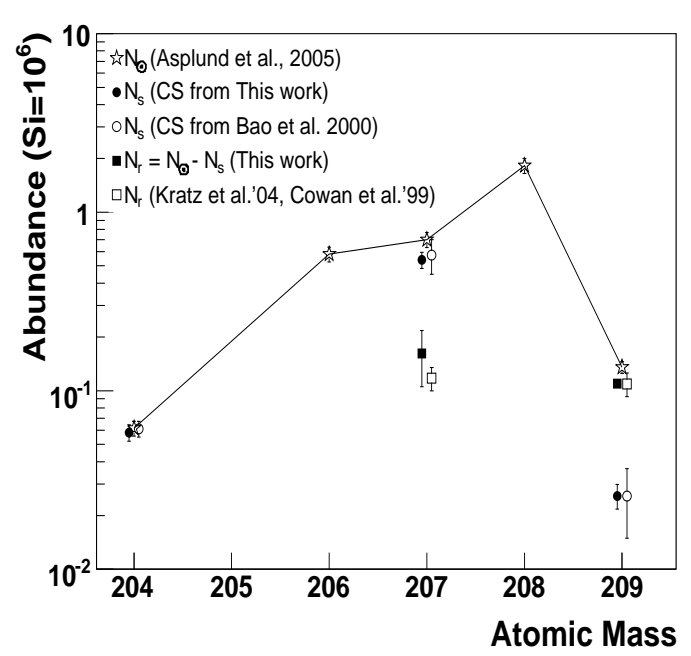

Figure 2: $s$-process solar abundances obtained with the MACS measured at n_TOF (solid circles) and with the MACS from Ref. [21] (open circles). Derived $r$-process residuals (solid squares) are compared versus $r$-process calculations $[23,24]$ (open squares).

In order to evaluate the impact of the new MACS and of their smaller uncertainties, calculations with a stellar model [25] were carried out. For ${ }^{204,207} \mathrm{~Pb}$ a calculation was made using two stellar masses, $M=1.5 M_{\odot}$ and $M=3 M_{\odot}$, and a combination of metallicities, $[\mathrm{Fe} / \mathrm{H}]=-0.3$ and $[\mathrm{Fe} / \mathrm{H}]=-1$, which have been found to account for the main and strong components [1,2], respectively. Since ${ }^{209} \mathrm{Bi}$ is mostly produced by the strong component, an AGB model with $M=3 M_{\odot}$ and $[\mathrm{Fe} / \mathrm{H}]=-1.3$ was used in that case. Our MACS for ${ }^{206} \mathrm{~Pb}$ was still preliminary (see previous section) and therefore no calculation with the new cross section has been made so far.

The impact of the more accurate MACS in the determination of the $s$-process abundances $N_{s}$ is best illustrated in Fig. 2. For ${ }^{207} \mathrm{~Pb}$ and ${ }^{209} \mathrm{Bi}$, the accuracy in the new s-process abundances (solid circles) could be improved by a factor of two when compared to the same abundance calculations based in previous cross sections from Ref. [21] (open circles). It can be concluded from the present experiment, that uncertainties in the $s$-process abundances of ${ }^{207} \mathrm{~Pb}$ and ${ }^{209} \mathrm{Bi}$ are now dominated by the uncertainties in the observed solar abundances (7-8\% for bismuth and 8-10\% for lead [26, $27,28]$ ) rather than by uncertainties in the nuclear data. In the case of the $s$-only isotope ${ }^{204} \mathrm{~Pb}$, its abundance uncertainty is still dominated by the uncertainties in the branching at ${ }^{204} \mathrm{Tl}$ [29].

By subtracting the new s-process abundances from the solar system abundances [28], more accurate $r$-process residuals could now be derived (solid squares in Fig. 2). These residuals can be compared versus detailed $r$-process calculations [23, 24] (open squares in Fig. 2). In the latter figure an uncertainty of $15 \%$ was considered for the $r$-process calculations [23]. Since ${ }^{207} \mathrm{~Pb}$ is mainly produced by the $s$-process, $N_{s}^{207}=77(8) \%$, the uncertainty resulting in the $r$-process residual is still considerably large $N_{r}^{207}=N_{\odot}^{207}-N_{s}^{207}=23(8) \%$. This result is in fair agreement with $r$-process abundance calculations, which yield values between 15\%[24] and 18\%[23] . The opposite situation occurs for bismuth, where the $s$-process abundance is smaller, leading to an accurately constrained 
$r$-process abundance $N_{r}^{209}=81(3) \%$. This result lies well within the 71-90\% abundance range predicted by $r$-process abundance calculations (see Fig. 2).

\section{Conclusions}

With the present measurements the neutron capture cross sections of the $\mathrm{Pb} / \mathrm{Bi}$ isotopes have been determined with improved accuracy. The respective improvements in the solar $s$-process abundances were determined with a set of stellar model calculations. With the new cross sections, the main uncertainty in the s-process abundances of ${ }^{207} \mathrm{~Pb}$ and ${ }^{209} \mathrm{Bi}$ is now due to the uncertainty of the solar abundances of lead and bismuth. On the other hand, the uncertainties of the branching at ${ }^{204} \mathrm{Tl}$ dominate the $s$-process abundance of ${ }^{204} \mathrm{~Pb}$. The $r$-process residuals derived from our results are in good agreement with $r$-process calculations.

\section{References}

[1] C. Travaglio et al., Astrophys. J. 521, 691 (1999).

[2] C. Travaglio et al., Astrophys. J. 549, 346 (2001).

[3] M. Heil et al., This Conference Proceedings.

[4] B.J. Allen et al., Phys. Rev. C 4, 1504 (1973).

[5] R. Plag et al., Nuclear Instruments and Methods in Physics Research A 496, 425 (2003).

[6] C. Domingo-Pardo et al., Phys. Rev. C 74, 025807 (2006).

[7] R. Macklin et al., Phys. Rev. C 14, 1389 (1976).

[8] P. Mutti et al., in proceedings of Nuclei in the Cosmos, edited by N. Prantzos and S. Harissopulos (Volos, Editions Frontières, 1998).

[9] PTB group (2002), n_TOF neutron fluence with the PTB Fission Chambers, CERN/SL/ETC/2002.

[10] R.L. Macklin et al., Nuclear Instruments and Methods in Physics Research A 164, 213 (1979).

[11] U. Abbondanno et al., Nuclear Instruments and Methods in Physics Research A 538, 692 (2005).

[12] R. Macklin et al., Phys. Rev. C 159, 1007 (1967).

[13] J.L. Tain et al., Jour. of Nucl. Sci. and Tech. Supl. 2, 689 (2002).

[14] U. Abbondanno et al., Nuclear Instruments and Methods in Physics Research A 521, 454 (2004).

[15] C. Domingo-Pardo et al., Phys. Rev. C (2006) in press.

[16] S. Raman et al., Physical Review Letters 39, 598 (1977).

[17] S. Raman et al., Physical Review Letters 40, 1306 (1978).

[18] M. Mizumoto et al., Phys. Rev. C 19, 335 (1979).

[19] L.R. Medsker et al., Phys. Rev. C 9, 709 (1973).

[20] N. Larson, SAMMY: Multilevel R-matrix fits to neutron data using Bayes equations, ORNL/TM-9179.

[21] Z.Y. Bao et al. (2000), At. Data Nucl. Data Tables 76,70.

[22] D.J. Horen et al., Phys. Rev. C 29, 2126 (1984). 
[23] J.J. Cowan et al., Astrophys. J. 521, 194 (1999).

[24] K.-L. Kratz et al., New Astronomy Review 48, 105 (2004).

[25] C. Arlandini et al., Astrophys. J. 525, 886 (1999).

[26] E. Anders et al., Geochimica et Cosmochimica Acta 53, 197 (1989).

[27] K. Lodders, Astrophys. J. 591, 1220 (2003).

[28] M. Asplund et al., ASP Conf. Ser. 336: Cosmic Abundances Records of Stellar Evolution and Nucleosynthesis, edited by T.G. Barnes, III and F.N. Bash (2005).

[29] U. Ratzel et al., Phys. Rev. C 70, 065803 (2004). 